\title{
Cardiomyocyte differentiation from iPS cells is delayed following knockout of Bcl-2
}

Tim Vervliet ${ }^{1 *}$, Robin Duelen ${ }^{2}$, H. Llewelyn Roderick ${ }^{3}$ and Maurilio Sampaolesi ${ }^{2}$

${ }^{1}$ Laboratory of Molecular and Cellular Signaling, Department of Cellular and Molecular Medicine, KU Leuven, 3000 Leuven, Belgium

${ }^{2}$ Translational Cardiomyology Laboratory, Department of Development and Regeneration, KU Leuven, 3000 Leuven, Belgium.

${ }^{3}$ Laboratory of Experimental Cardiology, Department of Cardiovascular Sciences, KU Leuven, 3000 Leuven, Belgium.

* Indicates corresponding author

Corresponding author:

Name: $\quad$ Tim Vervliet

Address: Laboratory of Molecular and Cellular Signaling

Department of Cellular and Molecular Medicine, KU Leuven

Campus Gasthuisberg, O\&N I Herestraat 49 - bus 802, 3000 Leuven, Belgium

Email: tim.vervliet@kuleuven.be 


\begin{abstract}
Anti-apoptotic B-cell lymphoma 2 ( $\mathrm{Bcl}-2)$ regulates a wide array of cellular functions involved in cell death, cell survival decisions and autophagy. $\mathrm{Bcl}-2$ acts by both direct interaction with different components of the pathways involved and by intervening in intracellular $\mathrm{Ca}^{2+}$ signalling. The function of $\mathrm{Bcl}-2$ is in turn regulated by post-translational modifications including phosphorylation at different sites by various kinases. Besides functions in cell death and apoptosis, $\mathrm{Bcl}-2$ regulates cell differentiation processes, including of cardiomyocytes, although the signalling pathways involved are not fully elucidated. To further address the role of $\mathrm{Bcl}-2$ during cardiomyocyte differentiation, we investigated the effect of its genetic knockout by CRISPR/Cas9 on the differentiation and functioning of human induced pluripotent stem cells to cardiomyocytes. Our results indicate that differentiation of iPS cells to cardiomyocytes is delayed by $\mathrm{Bcl}-2 \mathrm{KO}$, resulting in reduced size of spontaneously beating cells and reduced expression of cardiomyocyte $\mathrm{Ca}^{2+}$ toolkit and functionality. These data thus indicate that $\mathrm{Bcl}-2$ an essential protein for cardiomyocyte generation.
\end{abstract}




\section{Introduction}

B-cell lymphoma $2(\mathrm{Bcl}-2)$ is involved in the regulation of cell death and survival decisions at multiple levels. Canonically, the anti-apoptotic Bcl-2 protein binds to pro-apoptotic Bcl-2-family members, such as Bax and Bak, thereby inhibiting their pro-apoptotic functions as inducers of mitochondrial outer membrane permeabilization (MOMP) and thus cytochrome $\mathrm{C}$ release (1). In addition, $\mathrm{Bcl}-2$ has been shown to modulate inositol-1,4,5-trisphosphate receptors $\left(\mathrm{IP}_{3} \mathrm{R}\right)$ thereby inhibiting proapoptotic $\mathrm{Ca}^{2+}$ signalling towards the mitochondria (2). Other targets related to the cellular $\mathrm{Ca}^{2+}$ toolkit of $\mathrm{Bcl}-2$ include ryanodine receptors (RyR), sarco/endoplasmic reticulum ATPases and voltage dependent anion channels (3) Besides regulating the apoptotic outcome, Bcl-2 is involved in modulating autophagic flux. Specifically, Bcl-2 binds to Beclin 1 thereby limiting the initiation of autophagosome formation and thus autophagic flux (4).

Regulation of cell death and survival is also key during differentiation. Consistently, Bcl-2 is involved in the differentiation of certain cells and tissues. Neuronal differentiation for instance, has been shown to be regulated by $\mathrm{Bcl}-2$. Transplanting embryonic stem cells overexpressing $\mathrm{Bcl}-2$ in rat cortex enhanced recovery and neuronal differentiation after ischemic insult (5). In addition, overexpression of Bcl-2 in PC12 cells, neural crest tumour cells, led to increased expression of genes associated with neural differentiation whereas it decreased proliferation related genes (6). In the heart, Bcl-2 was shown to be upregulated in a GATA4-dependent manner (7), suggesting a role for Bcl-2 in cardiac development. Overexpression of $\mathrm{Bcl}-2$ in mouse heart resulted in increased proliferation of cardiomyocytes, also pointing towards a role for $\mathrm{Bcl}-2$ in heart development (8). The molecular pathways underlying $\mathrm{Bcl}-2$ regulation of cardiomyocyte differentiation remain elusive.

Phosphorylation of $\mathrm{Bcl}-2$ has been described to be involved in regulating $\mathrm{Bcl}-2$ functions. Several $\mathrm{Bcl}-$ 2 phosphorylation sites have been described in its unstructured loop, Thr56, Ser70 and Ser87, which have been shown to be targets of multiple kinases including mitogen-activated protein kinases (MAPK) (9-11). One of these kinases is the p38MAPK. Phosphorylation of Bcl-2 by p38MAPK was demonstrated to induce apoptosis by inhibiting the anti-apoptotic function of $\mathrm{Bcl}-2$ and inducing it to trigger the release of cytochrome $\mathrm{C}$ following MOMP induction in response to acute stressors (11). Phosphorylation of $\mathrm{Bcl}-2$ has also been shown to result in its translocation to the nucleus (12). In the nucleus, Bcl-2 interacts with c-Myc increasing its stability and transcriptional activity (13). Both p38MAPK and c-Myc have been reported to play critical roles in cardiomyocyte differentiation. p38MAPK commits mouse embryonic stem cells to differentiate into cardiomyocytes (14) and regulates cardiomyocyte proliferation (15). Cardiac c-Myc overexpression in mice increased cardiac mass and hyperplasia without having detrimental effects on cardiac myocyte maturation (16).

In this study, we aim to further identify the function of $\mathrm{Bcl}-2$ in cardiomyocyte differentiation. To this end, we analysed the effect of CRISPR/Cas9-mediated Bcl-2 knock-out (KO) on the cardiomyocyte differentiation of human induced pluripotent stem cells (hiPSC). We show that timely differentiation towards cardiomyocytes is dependent on $\mathrm{Bcl}-2$ as $\mathrm{Bcl}-2 \mathrm{KO}$ delayed this process. We further show that during cardiomyocyte differentiation $\mathrm{Bcl}-2$ is phosphorylated at Ser70 and that $\mathrm{Bcl}-2$ upregulation coincides with increased c-Myc expression. These results suggest that $\mathrm{Bcl}-2$ is critical for the correct generation of cardiomyocytes from iPS. 


\section{Materials and methods}

\section{Reagents and antibodies}

Unless otherwise specified, all chemicals were purchased from Merck. All primers and gene blocks used in this study were purchased from integrated DNA technologies (IDT; Leuven, Belgium). Primers were listed in (Supplemental table S1 and S2). Primary antibodies were anti-Vinculin, anti-cMyc, antiTubulin (Merck), anti-Bcl-2, anti-phospho Ser70-Bcl-2 (Santa Cruz), anti-phospho-p38MAPK, antip38MAPK, anti-Bcl-XL, anti-PARP (Cell Signalling), anti-LC3 (Nanotools), Anti-RyR (C3:33), anti-NCX (ThermoFisher), anti-cTnT (Abcam), anti-IP ${ }_{3}$ R2 (Abicode) and anti-SERCA2a (produced and gifted by Prof. F. Wuytack (KU Leuven).

\section{hiPSC culturing}

All experiments were performed using the commercially available Gibco ${ }^{\mathrm{TM}}$ Episomal hiPSC Line (A18945; Thermo Fisher Scientific). hiPSC were cultured feeder-free on Geltrex LDEV-Free hESCQualified Reduced Growth Factor Basement Membrane Matrix and maintained in Essential 8 Flex Basal Medium supplemented with Essential 8 Flex Supplement (50x) and 0.1\% Pen/Strep (all from Thermo Fisher Scientific), at $37^{\circ} \mathrm{C}$ under normoxic conditions $\left(21 \% \mathrm{O}_{2}\right.$ and $\left.5 \% \mathrm{CO}_{2}\right)$. Medium was changed daily. Colonies were routinely passaged non-enzymatically with $0.5 \mathrm{mM}$ EDTA in PhosphateBuffered Saline (PBS; both from Thermo Fisher Scientific).

\section{Cardiomyocyte differentiation of hiPSC}

For inducing cardiac differentiation, the PSC cardiomyocyte differentiation kit (Thermo Fisher Scientific) was used according to the manufacturers protocol. Briefly, prior to differentiation, hiPSC cells were seeded on a thin Matrigel Growth Factor Reduced (GFR) Basement Membrane Matrix layer and cultured for 3 or 4 days in Essential 8 medium at $37^{\circ} \mathrm{C}$ under hypoxic conditions $\left(5 \% \mathrm{O}_{2}\right.$ and $5 \%$ $\mathrm{CO}_{2}$ ) until the cells reached approximately $60 \%$ of confluency. Cardiomyocyte differentiation was started after the addition of a mesoderm inducing medium (medium A; day 0 ) for 48 hrs. After 24 $\mathrm{hrs}$, cells were transferred from hypoxia to normoxia. At day 2 of differentiation, cells were incubated for another $48 \mathrm{hrs}$ with a cardiomyocyte progenitor medium (medium B). From day 4 onwards, medium was changed every other day with a cardiomyocyte maintenance medium. For experimental purposes, samples were harvested via EDTA or Collagenase A (Merck) incubation and scraping on different days $(0,2,4,7,14$ and/or 21). All experiments performed were approved by the Research Ethical Committee of UZ/KU Leuven (protocol number S62524).

\section{Cloning of gRNA and selection plasmid}

The Bcl-2 gRNA (forward: 5'-GAGAACAGGGTACGATAACC-3' and reverse: 5'GGTTATCGTACCCtGTTCTC-3') was cloned in the pU6-(BbsI)CBh-Cas9-T2A-mCherry, a gift from Ralf Kuehn (Addgene plasmid \# 64324 ; http://n2t.net/addgene:64324 ; RRID:Addgene_64324) (17), using the $\mathrm{Bbsl}$ restriction enzyme. In order to introduce a hygromycin selection cassette into the genomic $B C L 2$ sequence, two genomic DNA sequences, in close proximity and on either side of a TTAA sequence within $500 \mathrm{bp}$ of the Bcl-2 gRNA, were identified. These sequences (one $430 \mathrm{bp}$ (HAL sequence (see 'Supplemental materials and methods') and a $450 \mathrm{bp}$ long (HAR sequence)) were introduced using the NEBuilder ${ }^{\circledR}$ HiFi DNA Assembly Master Mix (New England BioLabs) in combination with the sequences produced as gene blocks (IDT) and the PiggyBAC-Hygro-TK vector flanking the hygromycin resistance gene restricted with BamHI enzyme. In the HAR sequence, containing the start of the $B C L 2$ open reading frame, base pair substitution were incorporated in order to generate stop codons early in the $B C L 2$ gene resulting in the knock-out. 


\section{CRISPR/Cas9-mediated knock out of Bcl-2}

Nucleofection of the hiPSC was performed using the commercially available Cell Line Nucleofector Kit (Lonza) according to the manufacturers protocol. For the nucleofection, $2 \mu \mathrm{g}$ of the gRNA containing plasmid and $8 \mu \mathrm{g}$ of the selection plasmid were utilized. After nucleofection the hiPSC cells plated on Geltrex coated culture plates. On the third day after nucleofection, $50 \mathrm{ng} / \mathrm{ml}$ hygromycin was added to the medium to start the selection procedure. After 7 days of selection, single colony picking was performed. Every day the medium was refreshed with Essential 8 medium containing $50 \mathrm{ng} / \mathrm{ml}$ hygromycin until the colonies were sufficiently grown after which the cells were passaged. At this point a fraction of the hiPSC was taken for genomic DNA isolation to screen for the insertion of the hygromycin selection cassette at the correct locus in the BCL2 gene (see Supplemental table S1). Finally, the selection cassette was removed from the picked clones in which it was inserted at the exact intended location in the BCL2 gene. For this the hiPSC cells were detached and nucleofected with $5 \mu \mathrm{g}$ pf the piggyback transpose plasmid (kind gift of Dr. Catherine Verfaillie (KU Leuven)). The medium was changed daily using Essential 8 medium until cells reached $90 \%$ confluency. At this point Fialuridine (FIAU; Merck) selection was started for $24 \mathrm{hrs}$ to kill off the cells still containing the selection plasmid. After this the cells were allowed to recover and grow with daily medium changes followed by single colony picking a. Finally, a complete gene editing-free Bcl-2 KO hiPSC line was obtained due to PiggyBac excision and FIAU selection. Once the colonies were amplified, genomic DNA was collected in order to screen for the absence of the selection cassette, modification in the $B C L 2$ gene and potential off targets (see Supplemental table 1).

\section{Genomic DNA isolation}

Genomic DNA was isolated using a PureLink ${ }^{\mathrm{TM}}$ Genomic DNA Mini Kit (Invitrogen) according to the manufacturers protocol. The primers utilized for the subsequent PCR reactions can be found in Supplemental table S1. Sequencing of the PCR samples was performed by LGC genomics.

\section{Immunoblot analysis}

Cell pellets collected at different days of differentiation $(0,7,14$ and 21) were resuspended on ice in lysis buffer (20 mM Tris HCl, pH7.5, $150 \mathrm{mM} \mathrm{NaCl}, 1.5 \mathrm{mM} \mathrm{MgCl} 2,0.5 \mathrm{mM}$ DTT, $1 \%$ Triton X-100 and both protease (EDTA free protease inhibitors, Thermo Fisher Scientific) and phosphatase (PhosSTOP, Roche) inhibitors), followed by further homogenizing using an eppendorf douncer in order to facilitate lysis. Lysis was performed for at least $30 \mathrm{~min}$ with head over head mixing at $4^{\circ} \mathrm{C}$ followed by centrifugation for $5 \mathrm{~min}$ at $4^{\circ} \mathrm{C}$ at $>5000 \mathrm{xg}$. The supernatant was collected and protein concentrations were determined using a standard Bradford assay. Immunoblot samples were prepared at $0.5 \mu \mathrm{g} / \mu \mathrm{l}$ and were ran as described in (18). Following this primary antibody staining was performed over night at $4^{\circ} \mathrm{C}$ and secondary antibody staining with HRP conjugated antibodies (Bioke) was performed at room temperature for at least two hrs. Immunoblots were visualized using Pierce ${ }^{\mathrm{TM}}$ ECL Western Blotting Substrate (Thermo Fisher Scientific) in combination with a ChemiDoc ${ }^{\mathrm{TM}} \mathrm{MP}$ imaging system (BioRad).

\section{Quantitative reverse transcription PCR (RT-qPCR)}

RNA isolation was performed using a GenElute ${ }^{\mathrm{TM}}$ mammalian total RNA miniprep kit (Sigma) according to the manufacturers protocol. After this a DNA-free ${ }^{T M}$ kit (Invitrogen) was used according to the manufacturers protocols to remove potential contaminating DNA. RNA concentrations were determined using a Nanodrop. Using a high capacity cDNA reverse transcription kit (Applied Biosystems) CDNA was generated by reverse transcription of $1000 \mathrm{ng}$ total mRNA. Next, the qPCR reaction was set up. First, forward and reverse primers for the genes of interest (see Supplemental 
table S2) were mixed with FastStart Universal SYBR Green Master (Rox; Roche). $5 \mu$ of this mixture was pipetted in duplicate per condition in a 384 well plate. Next, the cDNA samples were diluted 10 times in nuclease free water and added to the primer FastStart mixture in the 384 wells. The ViiA7 Real-Time PCR System (Thermo Fisher Scientific) was used for performing the PCR reactions. For analysis, $\triangle \triangle C T$ values were determined for each condition after which the fold increase was calculated as $2^{-\triangle \Delta C T}$. As housekeeping genes GAPDH and RPL13a were utilized.

\section{Intracellular $\mathrm{Ca}^{2+}$ imaging}

Loading and de-esterification steps were performed in a humidified incubator at $37^{\circ} \mathrm{C}$ and $5 \% \mathrm{CO}_{2}$. hiPSC-derived cardiomyocytes differentiated for 9 or 11 days were incubated/loaded with $1 \mu \mathrm{M}$ Fluo4 AM solubilized in cardiomyocyte maintenance medium. Next, the cells were washed twice with cardiomyocyte maintenance medium after which de-esterification was allowed to occur for $45 \mathrm{~min}$. Just before starting the $\mathrm{Ca}^{2+}$ imaging experiments the maintenance medium was replaced with a prewarmed $\left(37^{\circ} \mathrm{C}\right)$ modified Krebs-Ringer solution $(135 \mathrm{mM} \mathrm{NaCl}, 6.2 \mathrm{mM} \mathrm{KCl}, 1.2 \mathrm{mM} \mathrm{MgCl}, 12 \mathrm{mM}$ HEPES, $\mathrm{pH} 7.3,11.5 \mathrm{mM}$ glucose and $2 \mathrm{mM} \mathrm{CaCl}_{2}$ ). Additions were performed as indicated in the figure. Tetracaine was solubilized in the above modified Krebs-Ringer solution at $1 \mathrm{mM}$ final concentration. Imaging was performed using a Nikon eclipse Ti2 inverted fluorescence microscope (Nikon) equipped with excitation filter FF01-378/474/554/635 and dichroic mirror FF01432/515/595/730 and emission filter 515/30 all from Semrock. Excitation was performed at $470 \mathrm{~nm}$ using a CoolLed pR-4000 (CoolLed). Acquisition of the fluorescent signal at $520 \mathrm{nM}$ was performed at $10 \mathrm{~Hz}$ using a pco.edge 4.2bi sCMOS camera (pCO). For image analysis, FIJI software (19) was utilized. The image stack were "resliced" across a straight line that was drawn from top to bottom over the largest responding area. The intensity profiles were plotted and measured as indicated in the figures. Traces are given as F/FO where the FO value was obtained after tetracaine administration. To determine the size of spontaneously responding areas, maximum intensity $Z$ projections were performed of the first 300 images per stack. A threshold for response was determined for one control condition in each experiment and applied to all other stack. After thresholding particle analyses was performed to determine the regions of interest from which the size of the areas were calculated.

\section{Statistical analysis}

Statistical analysis was performed using the GraphPad Prism software. For all conditions, normality assumptions were tested. If the data were normally distributed and the variability between the conditions were not significantly different from each other, one-way ANOVA tests with Tukey-post test for multiple comparison or one samples t-tests were performed. If not normally distributed the non-parametric Kruskall Wallis tests with Dunn's post-hoc multiple comparison were performed. More information on the performed statistical tests can be found in the figure legends. If not specified otherwise $p$ values $<0.05$ were considered significant (with $p<0.05, p<0.01, p<0.001$ and $p<0.0001$ are designated as $*, * *, * * *$ and $* * * *$ respectively). 


\section{Results}

\section{Creation and validation of $\mathrm{Bcl}-2 \mathrm{KO}$ hiPSC}

Bcl-2 KO was performed in the commercially available Gibco ${ }^{\mathrm{TM}}$ Episomal hiPSC Line (A18945) using a CRISPR/Cas9 based approach. After screening for their efficiency in HEK293 cells the most promising Bcl-2 gRNA, targeting an early sequence in the BCL2 gene, was selected and cloned in a vector coexpressing the Cas9 gene. The Bcl-2 gRNA plasmid was introduced into the hiPSC line together with a plasmid containing a hygromycin selection cassette flanked by DNA regions homologous to the Bcl-2 gene allowing the targeted introduction of the hygromycin cassette into the $B C L 2$ gene. Point mutations, resulting in early stop codons in the $B C L 2$ gene were introduced in one of the homologous regions, in order to introduce mutations in the $B C L 2$ gene. After antibiotic selection and single cell colony isolation, the insertion of the hygromycin cassette at the correct genomic locus was validated. Next, in three of the validated clones the hygromycin cassette was removed followed by FIAU selection, which kills cells containing the selection cassette. After single colony isolation, clones were screened for alterations in the $B C L 2$ gene. In figure 1 , we restricted the validation to the two clones, $\mathrm{Bcl}-2 \mathrm{KO} 1$ and $\mathrm{Bcl}-2 \mathrm{KO} 2$, used in this study. At the genomic level in all the tested clones, removal of the hygromycin cassette resulted in the base pair deletion or frame shift in the BCL2 gene. The majority of the tested clones showed a deletion of 4 base pairs (Figure $1 \mathrm{~A}$ ) resulting in the early introduction of three stop codons at the protein level (Figure 1B). When verified at the protein level via immunoblot, both clones showed absence of endogenous Bcl-2 (Figure 1C), suggesting a complete knock-out of the BCL2 gene. Finally, we screened for potential off-target effects of the used gRNA by sequencing the 10 most likely off targets of the gRNA in coding genes (Figure 1D). In both clones no alterations near the potential of target site were detected, suggesting that the $\mathrm{Bcl}-2 \mathrm{KO}$ occurred specifically in these cells and that these clones are suitable for use.

\section{$\mathrm{Bcl}-2 \mathrm{KO}$ does not inhibit but delays the induction of cardiac differentiation}

After validating and the selection of two Bcl-2 KO clones, we set out to determine the effects of $\mathrm{Bcl}-2$ $K O$ on cardiomyocyte differentiation. The differentiation scheme utilized in this study can be seen in Figure 2A. A commercially available cardiomyocyte differentiation kit, which utilizes three different types of medium for differentiation induction, was utilized and resulted in the robust production of cardiomyocyte cultures. A first observation in these cultures was that, as reported by the manufacturer, the clear spontaneous beating of large areas of cardiomyocytes could be observed as early as day 7 in the control differentiation. In the $\mathrm{BCl}-2 \mathrm{KO}$ clones, this only occurred at later days and was much more restricted in size (see also figure $4 \mathrm{~A}, \mathrm{~B}$ ). This indicates that $\mathrm{Bcl}-2 \mathrm{KO}$ may indeed impact cardiac differentiation at some level. To address this, we first assessed whether Bcl-2 KO resulted in impaired induction of early and late cardiomyocyte differentiation markers via RT-qPCR (Figure 2B). In control and $\mathrm{Bcl}-2 \mathrm{KO}$ conditions pluripotency markers decreased upon differentiation as expected. Mesoderm induction occurred as anticipated on day 2 of differentiation in both conditions. However, in the control condition there seems to be a trend towards more potent mesoderm induction. This trend could also be observed in the cardiac progenitor and late cardiac differentiation markers. This suggest that $\mathrm{Bcl}-2 \mathrm{KO}$ delays the differentiation of hiPSC to cardiomyocytes.

\section{$\mathrm{Bcl}-2 \mathrm{KO}$ does not trigger cell death induction or changes in autophagic flux in hiPSC-derived cardiomyocytes}

One explanation for the lower expression of cardiac progenitor and late cardiac differentiation markers in the $\mathrm{Bcl}-2 \mathrm{KO}$ conditions could be that $\mathrm{Bcl}-2 \mathrm{KO}$ results in loss of cells thus impacting 
differentiation in this manner. This is indeed plausible as Bcl-2 is known to play important functions in apoptosis and autophagy, two pathways which may control cell numbers.

$\mathrm{Bcl}-2$ is a key anti-apoptotic protein known to bind to and inhibit pro-apoptotic Bax and Bak, crucial for the execution of apoptosis. Therefore, $\mathrm{KO}$ of $\mathrm{Bcl}-2$ may indeed lead to increased apoptotic cell death. In order to address this, we performed immunoblot experiments and stained for poly ADPribose polymerase (PARP), which is cleaved by active caspase 3 an effector of apoptosis downstream of Bax/Bak activation. Quantification of the cleaved over total amount of PARP allows to assess caspase 3 activity which was unaltered in comparing Bcl-2 KO clones to control (Figure 3).

Bcl-2 also plays important roles in the regulation of autophagy. By binding to Beclin 1 for instance, $\mathrm{Bcl}-2$ impairs the induction of autophagosome formation thus inhibiting autophagic flux. In order to measure autophagy induction, we monitored the levels of lipidated microtubule-associated protein light chain 3 (LC3-II). No difference in LC3-II levels could be observed between control and BCl-2 KO clones (Figure 3), suggesting Bcl-2 KO did not alter basal autophagic flux induction.

Finally, we validated whether the $\mathrm{BCl}-2 \mathrm{KO}$ clones upregulated the closely related anti-apoptotic $\mathrm{Bcl}-$ $X_{L}$ protein to compensate for loss of $\mathrm{Bcl}-2$. However, $\mathrm{Bcl}-\mathrm{X}_{\mathrm{L}}$ levels were not significantly altered in the $\mathrm{Bcl}-2 \mathrm{KO}$ clones compared to the control (Figure 3).

\section{$\mathrm{Bcl}-2 \mathrm{KO}$ reduces the area of beating cells and inhibits the amplitude of spontaneous $\mathrm{Ca}^{2+}$ release in hiPSC-derived cardiomyocytes}

As the induction of cardiomyocyte differentiation seemed to be delayed upon $\mathrm{KO}$ of $\mathrm{Bcl}-2$ and no differences were observed in apoptosis and autophagy induction, we next set out to characterize in a more functional manner the effect of Bcl-2 KO in hiPSC-derived cardiomyocytes. For this, spontaneous $\mathrm{Ca}^{2+}$ release events, which are responsible for the spontaneous contractions, were measured. Intracellular $\mathrm{Ca}^{2+}$ imaging experiments were performed in cardiomyocytes differentiated for 9 or 11 days. At these time points it was feasible to detect spontaneously contracting areas in the $\mathrm{Bcl}-2 \mathrm{KO}$ cardiomyocytes whereas in the control line this was already observed on day 7 . The $\mathrm{Ca}^{2+}$ sensitive dye Fluo-4 AM was loaded in the cardiomyocytes after which spontaneous $\mathrm{Ca}^{2+}$ release was monitored using epi-fluorescence microscopy. Image acquisition was performed at $10 \mathrm{~Hz}$. First, Fluo4 loading allowed to determine the area of beating cells by monitoring spontaneous release. This is visualised in Figure $4 A$ as maximum intensity $Z$ projections of the first $30 \mathrm{sec}$ (300 images) of a control and the $\mathrm{BCl}-2 \mathrm{Ko}$ conditions. Quantification of the size of the spontaneously active area showed that in Bcl-2 KO cells this was severely decreased (Figure 4B). Next, we focused on the properties of the $\mathrm{Ca}^{2+}$ release. Quantification of each image stack was performed using the Fiji software by "reslicing" the stack over a vertical line drawn from top to bottom spanning the largest area of spontaneous activity in the stack (Figure 4C). The arrows in figure 4A show where these lines were drawn for "reslicing" these stacks respectively. From this line stack, intensity plots were determined and plotted as normalised fluorescence (F/FO). This was performed either for the entire slice area (black vertical bar Figure 4C) or was focussed on the area with the largest response (red bar, Figure 4C). A representative image of such a line stack for control and $\mathrm{Bcl}-2 \mathrm{KO}$ clones can be seen in figure $4 \mathrm{C}$ the intensity plot of the corresponding line stacks is shown in figure 4D. The analysis showed that compared to the control, $\mathrm{Bcl}-2 \mathrm{KO}$ did not alter the frequency of the spontaneous $\mathrm{Ca}^{2+}$ release events (Figure 4E). However, the amplitude (Figure 4F) and area under the curve (AUC; Figure 4G) were significantly lower in $\mathrm{BCl}-2 \mathrm{KO}$ cardiomyocytes compared to the control cardiomyocytes when quantifying the entire line stack. From figure $4 \mathrm{~A}, \mathrm{~B}$ and the line stack it is clear that a larger area of control cardiomyocytes exhibit spontaneous activity compared to the $\mathrm{Bcl}-2 \mathrm{KO}$ clones. We therefore also performed the same quantification but restricted to the major sites of response (red line Figure 
4C) and saw that both amplitude and AUC were no longer significantly different between control and $\mathrm{Bcl}-2 \mathrm{KO}$, although a trend was still present. These results confirm that $\mathrm{KO}$ of $\mathrm{BCl}-2$ does not impair but rather delays cardiomyocyte differentiation from hiPSC.

\section{$\mathrm{Bcl}-2 \mathrm{KO}$ impairs the expression of the cardiac $\mathrm{Ca}^{2+}$ toolkit}

Our findings suggest that $\mathrm{Bcl}-2 \mathrm{KO}$ delays differentiation to cardiomyocytes and this inhibits spontaneous $\mathrm{Ca}^{2+}$ release. Given the association between the maturation of the $\mathrm{Ca}^{2+}$ handling machinery involved in excitation contraction with cardiomyocyte maturation we thus investigated the underlying mechanisms for this reduced $\mathrm{Ca}^{2+}$ signalling capacity. To this end, immunoblot assays were performed to evaluate the expression levels of proteins involved in cardiac function and $\mathrm{Ca}^{2+}$ handling (Figure 5). From these immunoblots it is clear that the expression levels of RyR2, the sodium $\mathrm{Ca}^{2+}$ exchanger (NCX), the cardiac specific sarco/endoplasmic reticulum ATPase 2A (SERCA2a) isoform and cardiac Troponin T (cTnT) were dramatically lower in Bcl-2 KO cardiomyocytes compared to control cardiomyocytes. Inositol 1,4,5 Trisphosphate receptor type $2\left(I_{3} R 2\right)$ levels showed a similar decline in control and $\mathrm{Bcl}-2 \mathrm{KO}$ conditions. These results suggest that when examining the entire cell population in the $\mathrm{Bcl}-2 \mathrm{KO}$ cultures, only a minority is fully differentiated to cardiomyocytes. By focusing the $\mathrm{Ca}^{2+}$ imaging experiments on the spontaneously active regions we most likely also underestimate the effect of $\mathrm{Bcl}-2 \mathrm{KO}$ on cardiomyocyte differentiation

\section{Bcl-2 KO impairs early c-Myc upregulation thereby resulting in reduced functional cardiomyocyte growth}

$\mathrm{KO}$ of $\mathrm{BCl}-2$ did not alter the levels of apoptosis or autophagic flux. Besides functions in pathways involved in cellular removal, $\mathrm{Bcl}-2$ is also involved in the regulation of cellular proliferation differentiation and growth. Several MAPK, heavily involved in cell proliferation, are known to phosphorylate $\mathrm{Bcl}-2$, thereby regulating its function (11). Phosphorylation of $\mathrm{Bcl}-2$ by MAPK has been shown to lead to the nuclear translocation of $\mathrm{Bcl}-2$ where it provides pro-survival signalling and chemoresistance (12). In addition, nuclear Bcl-2 binds to c-Myc thereby regulating its stability and transcriptional activity. To validate whether this pathway is involved here we performed immunoblot staining (Figure 6A). First, the activity of p38MAPK was addressed by staining for its phosphorylated form and comparing it to the total p38MAPK levels. p38MAPK activity was not altered comparing control to $\mathrm{Bcl}-2 \mathrm{KO}$ conditions. However, during differentiation p38MAPK expression levels significantly increased in control cardiomyocytes, which was not the case in the $\mathrm{Bcl}-2 \mathrm{KO}$ cells. In addition, in the $\mathrm{Bcl}-2 \mathrm{KO} 1$ clone, p38MAPK levels appeared to be elevated prior to differentiation. Staining for phospho-Ser70 Bcl-2 revealed that in the control cardiomyocytes $\mathrm{Bcl}-2$ was phosphorylated at one of its the MAPK phosphorylation sites (Figure 6A). In the control cardiomyocytes, $\mathrm{Bcl}-2$ levels were upregulated during differentiation, confirming observations reported in (7). Finally, C-Myc expression levels were assessed (Figure 6B). Similar to the Bcl-2 levels, c-Myc expression was significantly elevated early in the differentiation of control but was lagging behind in the $\mathrm{Bcl}-2 \mathrm{KO}$ cardiomyocytes. These results suggest that $\mathrm{Bcl}-2 \mathrm{KO}$ results in reduced expression of c-Myc. As c-Myc is an important regulator of cardiomyocyte differentiation this may thus account at least in part for the delays observed in the $\mathrm{Bcl}-2 \mathrm{KO}$ conditions. 


\section{Discussion}

In this study, we show that $\mathrm{Bcl}-2$ plays an important part in regulating correct functional growth of cardiomyocytes derived from hiPSC. KO of Bcl-2 did not induce apoptosis or alter autophgic flux induction but instead resulted in delayed differentiation cardiomyocytes from hiPSC. Our result point towards a role for $\mathrm{Bcl}-2$ in regulating c-Myc stability, a transcription factor known to be required for cardiac differentiation and growth (16).

It is known that $\mathrm{Bcl}-2$ plays important roles during differentiation of neurons and cardiomyocytes however the pathways involved have not been fully elucidated yet (5-8). In this study we wanted to address this further and build on known functions of $\mathrm{BCl}-2$ in cardiomyocyte differentiation. To achieve this a CRISPR/Cas9-mediated knock-out of Bcl-2 was performed and validated in hiPSC (Figure 1). The CRSIPR/Cas9 assay that was setup in this study was intended to introduce specific point mutations via homologues recombination in the $B C L 2$ gene resulting in the introduction of stop codons early in the protein. Insertion of the selection cassette carrying the mutations was successful at the intended locus. However, after removal of the selection cassette none of the tested clones showed the introduction of the mutations. Instead the majority of the clones showed a 4 base pair deletion at the gRNA cut site resulting in a frame shift and insertion of three stop codons effectively knocking-out $\mathrm{Bcl}-2$. This was confirmed by immunoblot, which showed a complete $\mathrm{KO}$ of $\mathrm{BCl}-2$ compared to the control indicating that these clones were suitable for use in this study.

After validating the $\mathrm{KO}$ of $\mathrm{Bcl}-2$, the effects of lack of $\mathrm{Bcl}-2$ on cardiomyocyte differentiation were addressed. In our differentiations trend was observed suggesting a delay in the expression profile of markers associated with cardiomyocyte differentiation comparing control and $\mathrm{Bcl}-2 \mathrm{KO}$ (Figure 2). This was further confirmed by measuring spontaneous intracellular $\mathrm{Ca}^{2+}$ release, events as a measure of cardiomyocyte differentiation and maturation, in addition to measuring the size of areas spontaneous activity (Figure 4). From these experiments it was clear that control cardiomyocytes showed larger spontaneous $\mathrm{Ca}^{2+}$ release over larger areas compared to the $\mathrm{Bcl}-2 \mathrm{KO}$ conditions. These results further confirm that $\mathrm{Bcl}-2 \mathrm{KO}$ delays differentiation and thus limits the number of functional cardiomyocytes. A consideration here is that we are likely underestimating the effects of $\mathrm{BCl}-2 \mathrm{KO}$ on $\mathrm{Ca}^{2+}$ signalling. In these experiments, the decision to measure an area was made based on the presence of visual spontaneous activity. This was much more pronounced in the control cardiomyocytes compared to the $\mathrm{Bcl}-2 \mathrm{KO}$. As such in the $\mathrm{Bcl}-2 \mathrm{KO}$ we are likely sampling a sub population of the cells that actually did differentiate properly and as such also does not show large defects when specifically quantified.

The latter is further confirmed by the lack of expression of critical members of the cardiac $\mathrm{Ca}^{2+}$ toolkit in $\mathrm{Bcl}-2 \mathrm{KO}$ cardiomyocytes (Figure 5). These immunoblots monitor the average of the entire differentiating population. Based on the largely absent RyR, CTnT, SERCAa1 and NCX in the Bcl-2 KO condition one would not expect any spontaneous activity to be possible in the $\mathrm{Bcl}-2 \mathrm{KO}$ cardiomyocyte. Nevertheless, a subpopulation still was able to produce spontaneous $\mathrm{Ca}^{2+}$ release and contractions (Figure 4). Furthermore, Bcl-2 KO did not result in excessive induction of apoptosis or changes in autophagic flux (Figure 3). Taken together these results indicate that $\mathrm{Bcl}-2 \mathrm{KO}$ likely delays differentiation limiting the number of functional cardiomyocytes without affecting death of cardiomyocytes. This might be a more general function of $\mathrm{Bcl}-2$, not only restricted to cardiomyocytes, as $\mathrm{Bcl}-2 \mathrm{KO}$ mice have severe growth retardation which may be attributed to a general delay in differentiation when Bcl-2 is not present (20).

During cardiomyocyte differentiation both p38MAPK and c-Myc activity play important roles in differentiation and growth. p38MAPK has been shown to act as a switch deciding between 
proliferation and differentiation of cardiomyocytes $(14,15,21)$. c-Myc expression in cardiomyocytes on the other hand has been shown to be involved in cardiac growth (16). Bcl-2 has been shown to interact with nuclear c-Myc thereby regulating its transcriptional activity and stability (13). Bcl-2 translocation to the nucleus is dependent on its phosphorylation status (12). The p38MAPK phosphorylates $\mathrm{Bcl}-2$ and as such may facilitate its translocation towards the nucleus (11). Other MAPK are also known to phosphorylate Bcl-2. ERK1/2 for instance phosphorylates Bcl-2 at Ser70 thereby impacting Bcl-2 its anti-apoptotic functions (22). Importantly, phosphorylation of Bcl-2 at Ser70 by ERK1/2 was also shown to increase the functional cooperation between Bcl-2 and c-Myc (23). Our results show that during differentiation in both control and $\mathrm{BCl}-2 \mathrm{KO}$ conditions, p38MAPK is active (Figure $6 \mathrm{~A}$ ). In control condition, $\mathrm{Bcl}-2$ is phosphorylated and upregulated as has been previously described (7). Together with Bcl-2, c-Myc levels are upregulated early during the differentiation (Figure 6B). This upregulation of c-Myc is only observed at later time points in the Bcl$2 \mathrm{KO}$ cells which may at least in part explain the observed delay in cardiomyocyte differentiation (Figure 6B). These results are in agreement with literature that phosphorylation of $\mathrm{Bcl}-2$ by MAPK is linked to its functional cooperation with c-Myc (23).

In summary, our results indicate that Bcl-2 could be a link between MAPK and c-Myc signalling during early cardiomyocyte differentiation. Loss of $\mathrm{Bcl}-2$ impairs c-Myc expression resulting in a delayed differentiation of cardiomyocytes from hiPSC. 
bioRxiv preprint doi: https://doi.org/10.1101/2022.01.05.475068; this version posted January 5, 2022. The copyright holder for this preprint (which was not certified by peer review) is the author/funder. All rights reserved. No reuse allowed without permission.

\section{Acknowledgments}

This work was supported by the Research Foundation-Flanders (FWO) "krediet aan navorsers" (grant number: $1508319 \mathrm{~N})$. TV is a recipient of a senior post-doctoral grant of the FWO (grant number: $12 Z G 121 N)$

\section{Conflict of interest}

The authors declare no competing interests. 


\section{References}

1. Brunelle JK, Letai A. Control of mitochondrial apoptosis by the Bcl-2 family. J Cell Sci. 2009;122(4):437-41.

2. Rong YP, Aromolaran AS, Bultynck G, Zhong F, Li X, McColl K, et al. Targeting Bcl-2-IP 3 receptor interaction to reverse Bcl-2's inhibition of apoptotic calcium signals. Mol Cell. 2008;31(2):255-65.

3. Vervliet T, Parys JB, Bultynck $\mathrm{G}$. Bcl-2 proteins and calcium signaling: complexity beneath the surface. Oncogene. 2016;35(39):5079-92.

4. Pattingre S, Tassa A, Qu X, Garuti R, Liang XH, Mizushima N, et al. Bcl-2 antiapoptotic proteins inhibit Beclin 1-dependent autophagy. Cell. 2005;122(6):927-39.

5. Wei L, Cui L, Snider BJ, Rivkin M, Yu SS, Lee CS, et al. Transplantation of embryonic stem cells overexpressing $\mathrm{Bcl}-2$ promotes functional recovery after transient cerebral ischemia. Neurobiol Dis. 2005;19(1-2):183-93.

6. Liang Y, Mirnics ZK, Yan C, Nylander KD, Schor NF. Bcl-2 mediates induction of neural differentiation. Oncogene. 2003;22(35):5515-8.

7. Kobayashi S, Lackey T, Huang Y, Bisping E, Pu WT, Boxer LM, et al. Transcription factor gata4 regulates cardiac BCL2 gene expression in vitro and in vivo. FASEB J. 2006;20(6):800-2.

8. Limana F, Urbanek K, Chimenti S, Quaini F, Leri A, Kajstura J, et al. bcl-2 overexpression promotes myocyte proliferation. Proc Natl Acad Sci U S A. 2002;99(9):6257-62.

9. Maundrell K, Antonsson B, Magnenat E, Camps M, Muda M, Chabert C, et al. Bcl-2 undergoes phosphorylation by c-Jun $\mathrm{N}$-terminal kinase/stress-activated protein kinases in the presence of the constitutively active GTP-binding protein Rac1. J Biol Chem. 1997;272(40):25238-42.

10. Blagosklonny MV. Unwinding the loop of Bcl-2 phosphorylation. Leukemia. 2001;15(6):869-

74.

11. De Chiara G, Marcocci ME, Torcia M, Lucibello M, Rosini P, Bonini P, et al. Bcl-2

Phosphorylation by p38 MAPK: identification of target sites and biologic consequences. J Biol Chem. 2006;281(30):21353-61.

12. Zhou M, Zhang Q, Zhao J, Liao M, Wen S, Yang M. Phosphorylation of Bcl-2 plays an important role in glycochenodeoxycholate-induced survival and chemoresistance in HCC. Oncol Rep. 2017;38(3):1742-50.

13. Jin Z, May WS, Gao F, Flagg T, Deng X. Bcl2 suppresses DNA repair by enhancing c-Myc transcriptional activity. J Biol Chem. 2006;281(20):14446-56.

14. Aouadi M, Bost F, Caron L, Laurent K, Le Marchand Brustel Y, Binetruy B. p38 mitogenactivated protein kinase activity commits embryonic stem cells to either neurogenesis or cardiomyogenesis. Stem Cells. 2006;24(5):1399-406.

15. Engel FB, Schebesta M, Duong MT, Lu G, Ren S, Madwed JB, et al. p38 MAP kinase inhibition enables proliferation of adult mammalian cardiomyocytes. Genes Dev. 2005;19(10):1175-87.

16. Jackson T, Allard MF, Sreenan CM, Doss LK, Bishop SP, Swain JL. The c-myc proto-oncogene regulates cardiac development in transgenic mice. Mol Cell Biol. 1990;10(7):3709-16.

17. Chu VT, Weber T, Wefers B, Wurst W, Sander S, Rajewsky K, et al. Increasing the efficiency of homology-directed repair for CRISPR-Cas9-induced precise gene editing in mammalian cells. Nat Biotechnol. 2015;33(5):543-8.

18. Vervliet T, Decrock E, Molgo J, Sorrentino V, Missiaen L, Leybaert L, et al. Bcl-2 binds to and inhibits ryanodine receptors. J Cell Sci. 2014;127(Pt 12):2782-92.

19. Rueden CT, Schindelin J, Hiner MC, DeZonia BE, Walter AE, Arena ET, et al. ImageJ2: ImageJ for the next generation of scientific image data. BMC Bioinformatics. 2017;18(1):529.

20. Veis DJ, Sorenson CM, Shutter JR, Korsmeyer SJ. Bcl-2-deficient mice demonstrate fulminant lymphoid apoptosis, polycystic kidneys, and hypopigmented hair. Cell. 1993;75(2):229-40.

21. Romero-Becerra R, Santamans AM, Folgueira C, Sabio G. p38 MAPK Pathway in the Heart: New Insights in Health and Disease. Int J Mol Sci. 2020;21(19). 
22. Deng X, Ruvolo P, Carr B, May WS, Jr. Survival function of ERK1/2 as IL-3-activated, staurosporine-resistant Bcl2 kinases. Proc Natl Acad Sci U S A. 2000;97(4):1578-83.

23. Jin Z, Gao F, Flagg T, Deng X. Tobacco-specific nitrosamine 4-(methylnitrosamino)-1-(3pyridyl)-1-butanone promotes functional cooperation of Bcl2 and c-Myc through phosphorylation in regulating cell survival and proliferation. J Biol Chem. 2004;279(38):40209-19. 


\section{Figure legends:}

\section{Figure 1: CRISPR/Cas9-mediated KO of Bcl-2 in hiPSC}

A) Sequencing result of the first 100 base pairs of the genomic $\mathrm{Bcl}-2$ open reading frame comparing the sequence of the control condition to the two Bcl-2 KO hiPSC clones utilized in this study. The utilized gRNA is indicated by the black box in the BCL2 reference sequence (ref seq.). B) Predicted protein sequence based on the sequencing result obtained in $\mathrm{A}$ for the control and Bcl-2 KO hiPSC. * indicates the presence of a stop codon. C) Immunoblot validating the absence of Bcl-2 in the Bcl-2 KO clones compared to the control. D) Sequencing result surrounding the 10 most likely off target genes associated with the utilized gRNA. The potential off target is indicated by the black box in the ref seq. All DNA sequences and profiles were visualized using SnapGene ${ }^{\circledR}$ software

\section{Figure 2: $\mathrm{BCl}-2 \mathrm{KO}$ does not impair the expression of cardiac differentiation markers}

A) Scheme of the utilized cardiac differentiation protocol and time points where samples were harvested or utilized for intracellular $\mathrm{Ca}^{2+}$ imaging. B) RT-qPCR analysis of control and the two Bcl-2 KO clones screening for typical gene expression profile associated with cardiomyocyte differentiation at different time points $(0,2,4,714,21$ days of differentiation). The data points represent average fold increase $\left(2^{-\Delta \Delta C T}\right) \pm$ SEM of at least 3 different differentiations $(n \geq 3)$ of the indicated genes at the indicated timepoints. ANOVA tests with Tukey-post test for multiple comparison were performed * indicates $\mathrm{p}>0.05$ and ${ }^{* *}$ indicates $\mathrm{p}>0.01$.

\section{Figure 3: $\mathrm{KO}$ of $\mathrm{Bcl}-2$ does not induce apoptosis or changes in autophagic flux}

Left, immunoblot analysis of cardiomyocytes derived from control or $\mathrm{Bcl}-2 \mathrm{KO}$ clones differentiated for $0,7,14$ or 21 days, stained for the indicated proteins. Right, quantification of the performed immunoblot experiments. For PARP cleavage, the expression levels of cleaved PARP were divided by the total PARP levels. All other protein expression levels are plotted normalized to their corresponding loading control (vinculin) and relative to the levels for control day 0 . Each data point represents and independent repetition or differentiation. All differentiations were performed at least 4 times $(n \geq 4)$. The assumptions of normality were met and therefore parametric tests were performed. For comparing day 0 values, one sample t-tests were performed to compare to the normalized control value of control day 0 . For all other days, one-way ANOVA tests with Tukey-post test for multiple comparison were performed, * indicate $p<0.05$.

Figure 4: $\mathrm{KO}$ of $\mathrm{Bcl}-2$ reduces the area of spontaneous activity as well as the spontaneous $\mathrm{Ca}^{2+}$ release

Spontaneous intracellular $\mathrm{Ca}^{2+}$ measurements in cardiomyocytes differentiated for 9 or 11 days using Fluo-4. A) Maximum intensity plots of the first $30 \mathrm{sec}$ (300 images) of a representative $\mathrm{Ca}^{2+}$ measurement for control and $\mathrm{Bcl}-2 \mathrm{KO}$ conditions. The arrows indicate where the line was drawn for "reslicing" the stack in C. B) Quantification of the area of spontaneous activity derived from the maximum intensity plots. Each data point represents the size of the area of spontaneous activity of a single $\mathrm{Ca}^{2+}$ imaging experiments. Each experimental day, values were put relative to the control condition with the smallest area of spontaneous activity $(n \geq 9)$. C) Visual representation of the experiment showing a time laps of the line scans obtained after re-slicing the image stack for control and $\mathrm{Bcl}-2 \mathrm{KO}$ conditions. After $60 \mathrm{sec}$, tetracaine $(1 \mathrm{mM})$ was added to block RyR activity and determine the baseline $\mathbf{D}$ ) Intensity profiles obtained by quantifying the entire area (indicated by the black bar left of the line scans in A) plotted as F/FO. FO was determined after tetracaine addition. 
Quantification of the spontaneous activity was obtained by determining the frequency $\mathbf{E}$, amplitude $\mathbf{F}$ and area under the curve $\mathbf{G}$. For amplitude and frequency an additional quantification was performed focussing only on the areas of most intense responses (indicated by the red bar left of the line scans in $\mathbf{C}$ ). All experiments were performed at least 6 times $(n \geq 6)$. For statistical analysis oneway ANOVA tests with Tukey-post test for multiple comparison were performed.

\section{Figure 5: $\mathrm{KO}$ off $\mathrm{Bcl}-2$ does impairs the expression of the cardiac $\mathrm{Ca}^{2+}$ toolkit}

Left, Immunoblot analysis of cardiomyocytes derived from control or Bcl-2 KO clones differentiated for $0,7,14$ or 21 days, stained for the indicated proteins. Right, quantification of the performed immunoblot experiments. All protein expression levels are normalized towards their corresponding loading control (tubulin). On each blot all values were normalized to a control condition where expression levels were easily detected. For RyR, SERCA2a, CTNT, NCX this was control day 14 as for $I_{3} R 2$ this was control day 0 . Each data point represents and independent differentiation which were performed at least 4 times $(n \geq 4)$. As the assumption for normal distribution were not met for all conditions, non-parametric Kruskall-Wallis tests with Dunn/s multiple comparison were performed * indicates $p<0.05$ and ${ }^{* *}$ indicates $p<0.01$.

\section{Figure 6: Bcl-2 KO impairs upregulation of c-Myc}

A,B) Left, immunoblot analysis of cardiomyocytes derived from control or $\mathrm{Bcl}-2 \mathrm{KO}$ clones differentiated for $0,7,14$ or 21 days, stained for the indicated proteins. Right, quantification of the performed immunoblot experiments. For quantifying phosphorylated p38MAPK or phosphorylated $\mathrm{BCl}-2$, the protein levels detected by the phospho-antibody were normalized to the total amount of p38MAPK or Bcl-2. For the other quantifications protein levels were normalized to their respective loading control (vinculin) and put relative to a control condition were the protein was easily detected. For p38MAPK this was control day 0 as for c-Myc this was control day 14. All differentiations (represented by a data point) were performed at least times $(n \geq 3)$. The assumptions for normal distribution were met for all Bcl-2 KO conditions therefore one-way ANOVA tests with Tukey-post test for multiple comparison were performed for all these conditions. In the control samples, the conditions used to normalize towards do not have any variability nor are normally distributed therefore, in this case Kruskall-Wallis tests with Dunn/s multiple comparison were performed * indicates $p<0.05$ 
Bcl-2 Genomic DNA

Ref seq. atggcgcacgctg gagaacaggtacgataacdgggagatagtgatgaagtacatccattataagctgtcgcagaggggctacgagtgggatgcg ATGGCGCACGCTGGGAGAACAGGGTACGATAACCGGGAGATAGTGATGAAGTACATCCATTATAAGCTGTCGCAGAGGGGCTACGAGTGGGATGCG

Control

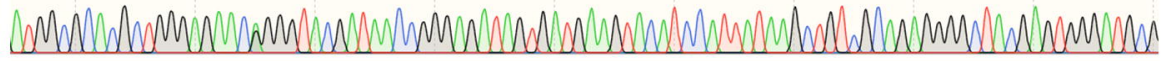

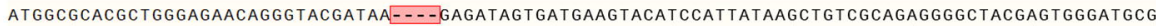

Bcl-2 KO1

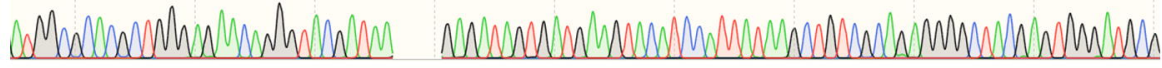

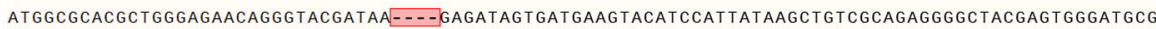

BCl-2 KO2

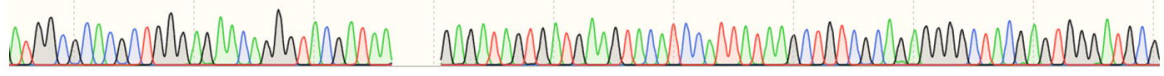

\section{B}

\section{$\mathrm{Bcl}-2$ protein sequence}

Control MAHAGRTGYDNREIVMKYIHYKLSQRGYEWDAGDVGAAPPGAAPAPGIFSSQPGHTPHPA Bcl-2 KO1 MAHAGRTGYDKR ***STSI---ISCR-----RGATSGMREMWAPRPR------GPPPHRA

Bcl-2 KO2 MAHAGRTGYDKR ***STSI---ISCR-----RGATSGMREMWAPRPR-------GPPPHRA

\section{D}

\section{OT1: RBFOX3}

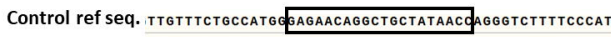
TTGTTTCTGCCATGGGAGAACAGGCTGCTATAACCAGGGTCTTTTCCCAT

Bcl-2 KO1

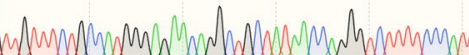
Bcl-2 KO2 tTGTtTCtgccatgggagaAcaggctgctataAccaggGtctittcccat

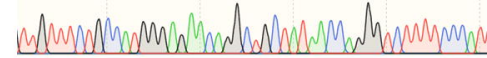

\section{OT2: SLC2A9}

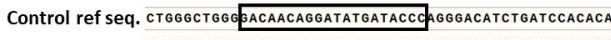
CTGGGCTGGGGACAACAGGATATGATACCCAGgGACATCTGATCCACACA

Bcl-2 KO1

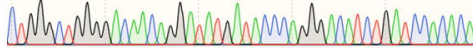

Bcl-2 KO2

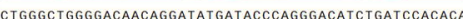

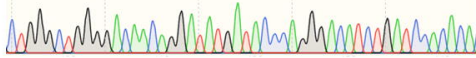

\section{OT3: ANKRD}

Control ref seq. GGGTACTTTGAGAATCAG GAGACCAGGGTACTCTCAC QAGGTGTTTGTGA GGGTACTITGAGAATCAGGAGACCAGGG TACTCTCACCAGGTGITTGTGA

Bcl-2 KO1

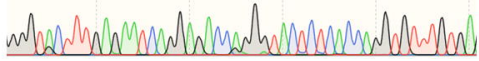

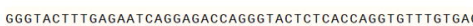
$\mathrm{Bcl}-2$ KO2

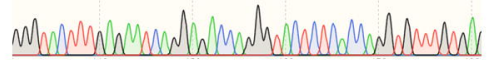

\section{OT4: NEFL}

Control ref seq. TAAAACc GAGAACATGGTACAATAAGA GGAATAATITTTATCTATAT TAAAACCTGAGAACATGGTACAATAAGAAGGATAATTITTTATCTATAT

Bcl-2 KO1

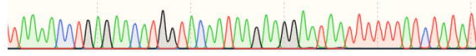
TAAAACCTGAGAACATGG TACAATAAGAagGaATAATTIUITATCTATAT,

$\mathrm{Bcl}-2 \mathrm{KO2}$

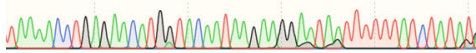

OT5: SLC23A3

Control ref seq. TGITATTACC STGAAAGGGTATGATCACC TGGAACCCAAGGACTCTTC tGITATTACCTGTGAAAGGGTATGATCACC TGGAACCCAAGGACTCTTC

Bcl-2 KO1

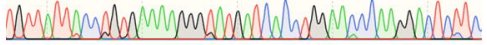
tgITATTACC TGTGAAAAGGG TATGATCACC TGGAACCCAAGGACTCTTC
C

Anti-Vinculin

Anti-Bcl-2

\section{OT6: PTPRT}

Control ref seq. CAAGCCAGGCTGTGGTAACCTGTTCT AGTGTCCCGGAatTACATGACCT CAAGCCAGGCTGTGGTAACCTGTTCTCAGTGTCCCGGAATTACATGACCT

Bcl-2 KO1

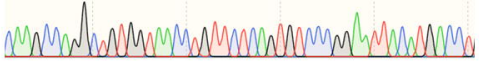

CAAGCCAGGCTGTGGTAACCTGTTCTCAGTGTCCCGGAATTACATGACCT

$\mathrm{Bcl}-2 \mathrm{KO} 2$

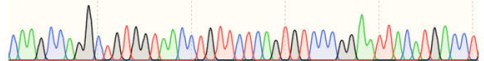

OT7: NCKAP5

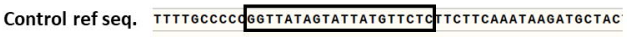

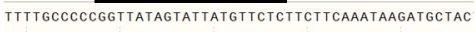

Bcl-2 KO1

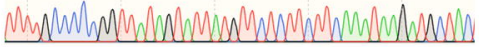

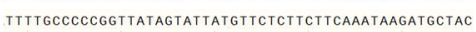

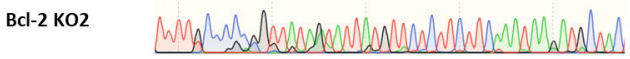

OT8: FRAS1

Control ref seq. cCACAAACC $\triangle$ ATTATCGTTCCTTGTTCT CCCTCCCTGGCAGAGGCAGG CCACAAACCAAGTTATCGTTCCTTGTTCTICCCTCCCTGGCAGAGGCAGG

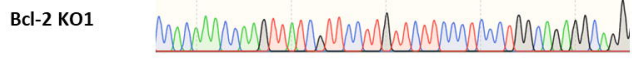
CCACAAACCAAGITATCGTTCCITGTTCTTCCCTCCCTGGCAGAGgCAgO

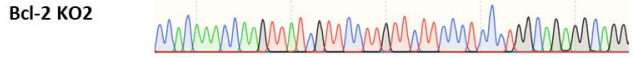
OT9: DLG2

Control ref seq. IAGGTTGTTC AAGAACAGGGTAGGATTATCRGGAGTGTTGAGCCTTTT

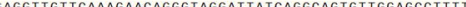

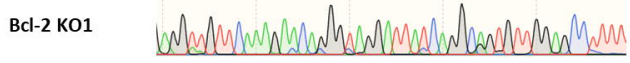
iAgGTTGTTCAAAGAACAGGGTAGGATTATCAGGCAGTGTTGGAGCTTTT

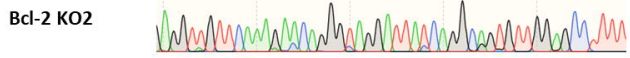

OT10: PCDH19

Control ref seq. TAGTCATAGg GAGACCAGGGTAGGAGACG GGCTGAGTCAATGTAAAA tagtcatagggagaccaggGtaAgagaACGTGgCtgagtcaAtgtaAaA:

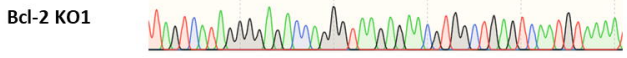
TAGTCATAGGGGAGACCAGGGTAAGAGAACGTGGCTGAGTCAATGTAAAA

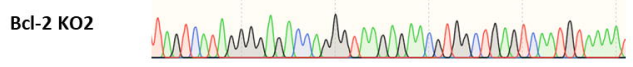




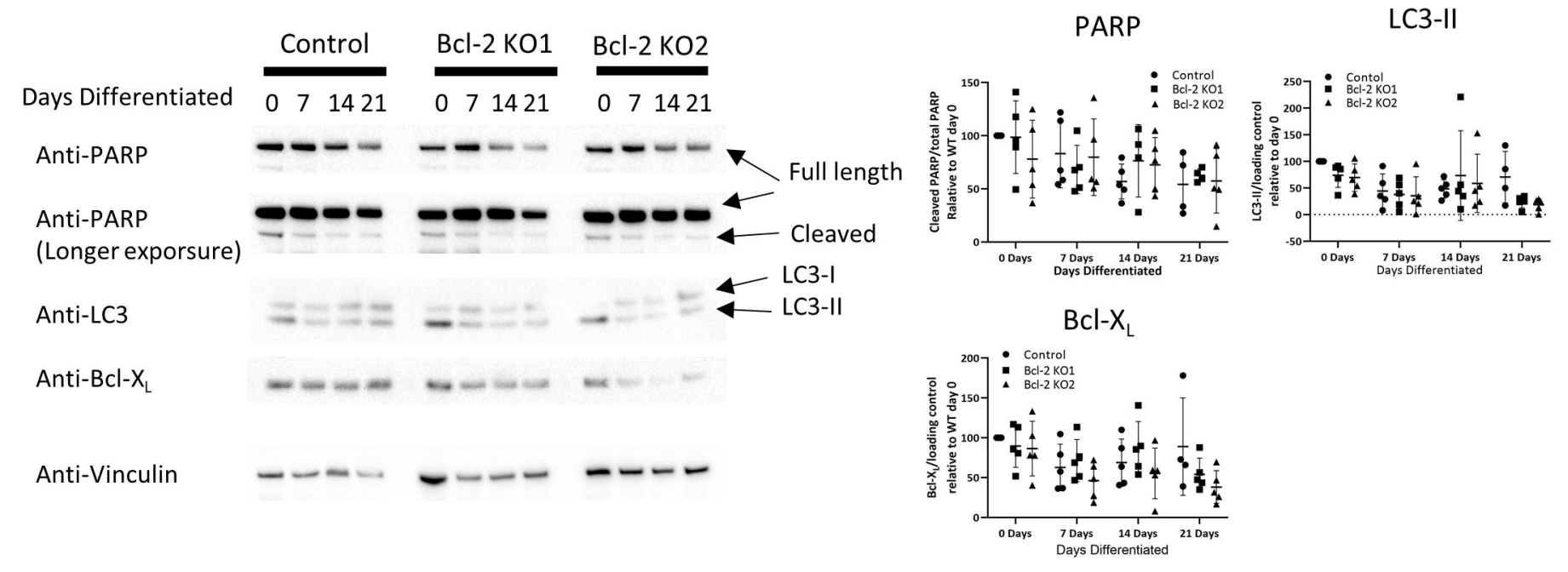

Figure 3 


\section{RyR NCX}

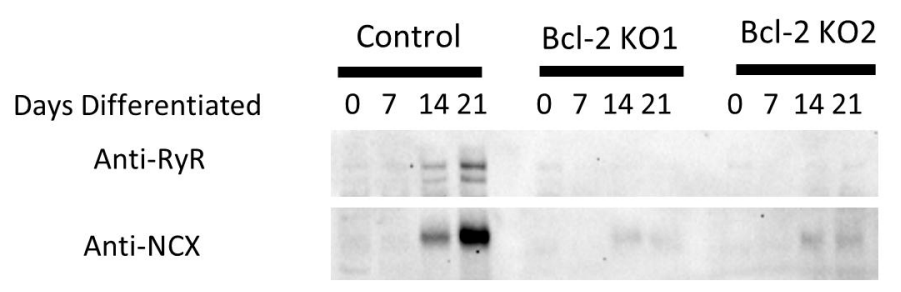
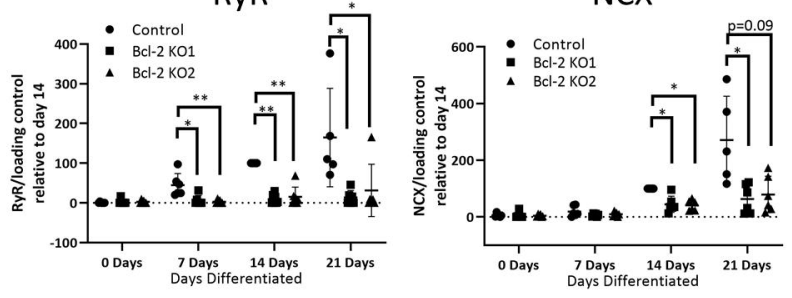

\section{Anti-cTnT}

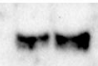

Anti-Tubulin

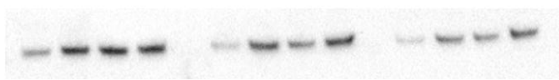

cTnT

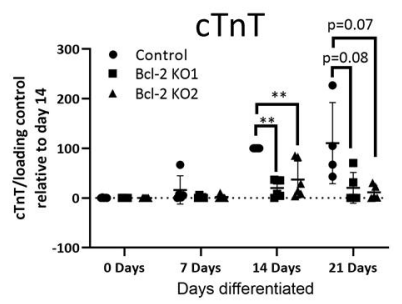

$\mathrm{IP}_{3} \mathrm{R} 2$
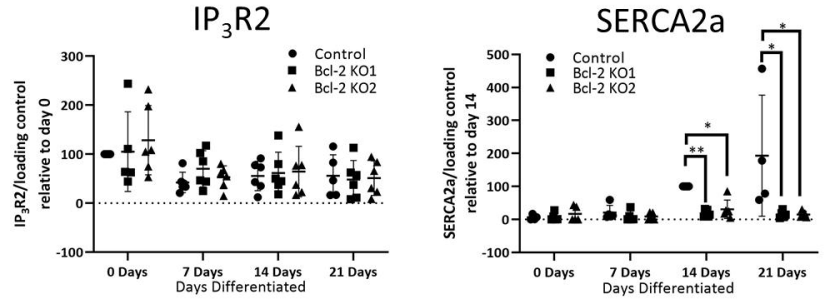

Figure 5 
A

Days Differentiated

Anti-phosphop38MAPK

Anti-p38MAPK

Anti-phospho-

Ser70 Bcl-2

Anti-Bcl-2

Anti-Vinculin

\section{Control

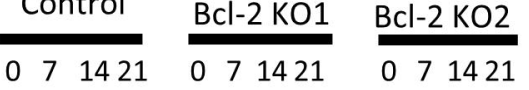

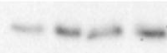

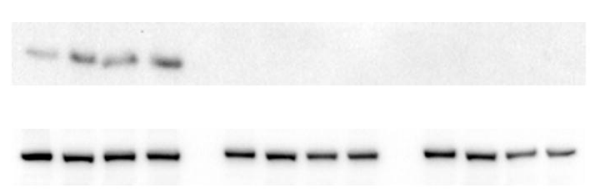

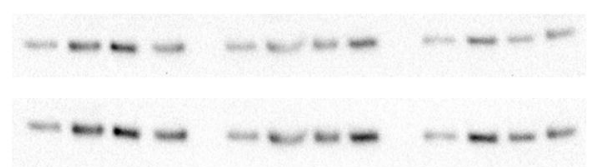

Control

$\mathrm{Bcl}-2 \mathrm{KO} 1 \mathrm{Bcl}-2 \mathrm{KO} 2$

$\begin{array}{lllllllllllll}0 & 7 & 1421 & \mathrm{~L} & 0 & 7 & 1421 & \mathrm{~L} & 0 & 7 & 1421\end{array}$

B

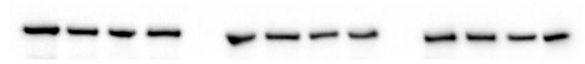

Phospho-

p38MAPK

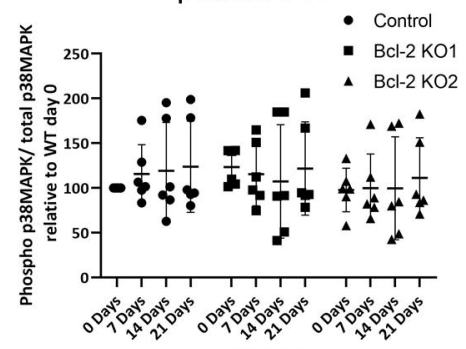

Days Differentiated

Phospho-Bcl-2

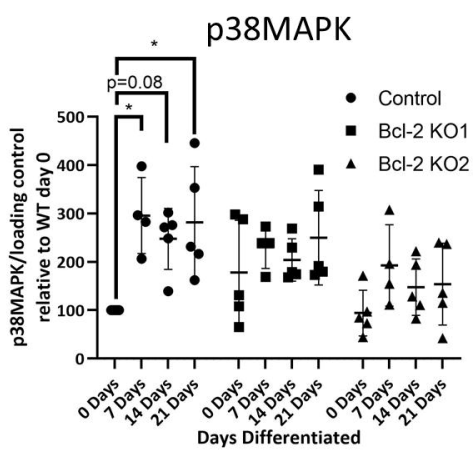

$\mathrm{Bcl}-2$
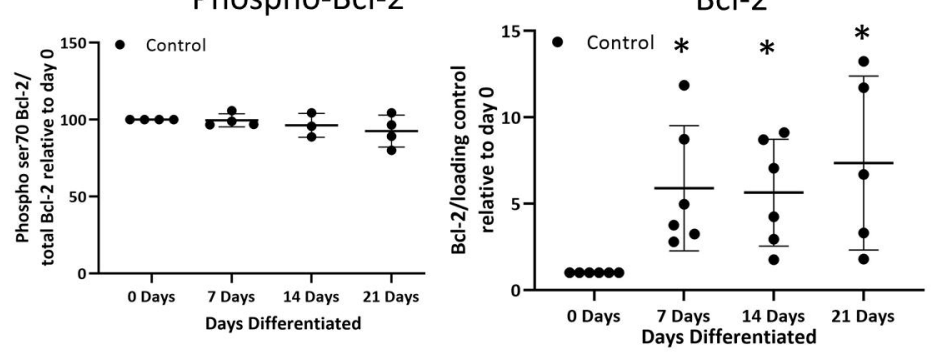

Anti-c-Myc

Anti-Vinculin

Days Differentiated

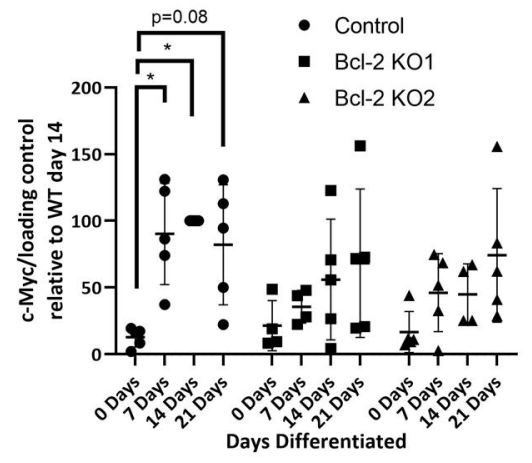

Figure 6 